\title{
Comparison of risk factors for squamous cell and adenocarcinomas of the cervix: a meta-analysis
}

\author{
A Berrington de González*,', S Sweetland' and J Green' \\ 'Cancer Research UK Epidemiology Unit, Gibson Building, Radcliffe Infirmary, Woodstock Road, Oxford OX2 6HE, UK
}

\begin{abstract}
While most cancers of the uterine cervix are squamous cell carcinomas, the relative and absolute incidence of adenocarcinoma of the uterine cervix has risen in recent years. It is not clear to what extent risk factors identified for squamous cell carcinoma of the cervix are shared by cervical adenocarcinomas. We used data from six case-control studies to compare directly risk factors for cervical adenocarcinoma ( 910 cases) and squamous cell carcinoma (5649 cases) in a published data meta-analysis. The summary odds ratios and tests for differences between these summaries for the two histological types were estimated using empirically weighted least squares. A higher lifetime number of sexual partners, earlier age at first intercourse, higher parity and long duration of oral contraceptive use were risk factors for both histological types. Current smoking was associated with a significantly increased risk of squamous cell carcinoma, with a summary odds ratio of 1.47 (95\% confidence interval: 1.15-1.88), but not of adenocarcinoma (summary odds ratio $=0.82(0.60-1.11)$; test for heterogeneity between squamous cell and adenocarcinoma for current smoking: $P=0.001)$. The results of this meta-analysis of published data suggest that squamous cell and adenocarcinomas of the uterine cervix, while sharing many risk factors, may differ in relation to smoking. Further evidence is needed to confirm this in view of the limited data available.
\end{abstract}

British Journal of Cancer (2004) 90, |787-179I. doi:I0.1038/sj.bjc.660I764 www.bjcancer.com

Published online 13 April 2004

(C) 2004 Cancer Research UK

Keywords: cervix neoplasms; risk factors; adenocarcinoma; squamous cell carcinoma; smoking

Most cancers of the uterine cervix are squamous cell carcinomas, but the relative and absolute incidence of adenocarcinoma has risen in recent years and adenocarcinomas now account for about $20 \%$ of incident invasive cervical cancers in screened populations worldwide (Sasieni and Adams, 2001). It remains unclear to what extent risk factors identified for squamous cell carcinoma of the cervix are shared by cervical adenocarcinomas (Parazzini and La Vecchia, 1990; Kjaer and Brinton, 1993; Altekruse et al, 2003; Green et al, 2003). While infection with the human papillomavirus (HPV) appears to be the most important cause of both types of cervical cancer (Walboomers et al, 1999; Clifford et al, 2003), some controlled studies have found differences between adenocarcinoma and squamous cell carcinoma in the importance of other factors such as smoking (Lacey et al, 2001; Green et al, 2003) and reproductive factors (Altekruse et al, 2003). Individual studies have generally been limited by small numbers of adenocarcinoma cases and in some instances by lack of adjustment for confounding factors. In the 10 years since this subject was last reviewed (Parazzini and La Vecchia, 1990; Kjaer and Brinton, 1993), a number of new studies have been published. In this meta-analysis of published data, we have combined results from those controlled studies that provided a direct comparison between risk factors for squamous cell and adenocarcinoma, to assess the current evidence.

\footnotetext{
*Correspondence: Dr A Berrington de González;

E-mail: amy.berrington@cancer.org.uk

Received 28 January 2004; accepted 6 February 2004; published online 13 April 2004
}

\section{MATERIALS AND METHODS}

Studies were identified through searches of MEDLINE (1966-June 2003, using combinations of the search terms 'cervix neoplasms', 'risk factors', 'adenocarcinoma' and 'squamous cell carcinoma') and of bibliographies of identified papers. We included any controlled study that provided the age-adjusted odds ratios and 95\% confidence intervals (CIs) for both adenocarcinoma (including adenosquamous carcinoma) and squamous cell carcinoma of the cervix (invasive or in situ) for at least one of the following risk factors (but not necessarily in the same publication): duration of oral contraceptive use, smoking, reproductive factors and sexual behaviour. Studies providing information on only one of the two histological types were not included, to ensure that any potential differences between the types were not due to study design or setting. No limit was placed on the number of cases. The most adjusted odds ratio available was used for analysis. In most studies, oral contraceptive use was not further defined and may include combined and progestagen-only oral contraceptives; however, the large majority of oral contraceptive users in these studies are likely to have used combined preparations (IARC, 1999).

\section{Statistical methods}

The odds ratios from each study were grouped into the closest of the prespecified categories for each risk factor (e.g. for duration of oral contraceptive use $<5,5-9$ and $10+$ years). To enable the results for the studies that had been divided into more categories to be included, it was necessary to combine some of the categories 
using a method for combining nonindependent strata (Berrington and Cox, 2003).

The summary (odds ratios, OR) for the pooled data were calculated under a fixed effects model using the method of empirically weighted least squares, where the weights are defined as the inverse of the variance of the log odds ratios (Cox and Snell, 1989). Heterogeneity between individual study results and between summary risk estimates for the two histological types was also calculated using this method.

In Figure 1, summary OR for groups of studies are shown as black circles whose size does not represent the amount of data available. In Figure 2, OR for individual studies are plotted as black squares whose size is inversely proportional to the variance of the logarithm of the odds ratios diamonds represent the summary odds ratios with $95 \%$ CIs indicated by their horizontal extent.

\section{RESULTS}

Data were available from six case-control studies: by Brinton and co-workers in the USA (Brinton et al, 1986) and Latin America (Brinton et al, 1990, 1993); the World Health Organisation (WHO) multicentre study (WHO, 1985; Thomas and Ray, 1996); a multicentre study by Lacey and co-workers in the USA (Lacey et al, 1999, 2001; Altekruse et al, 2003); a pooled analysis from the International Agency for Research on Cancer (IARC) (Munoz et al, 2002; Plummer et al, 2003) of data from 10 individual studies, of which two (Chichareon et al, 1998; Ngelangel et al, 1998) were included individually in analyses for which the pooled IARC data were not available; and the UK National Case-Control Study of
Cervical Cancer (Green et al, 2003). In total, data were available for 5649 cases of squamous cell carcinoma, 910 cases of adenocarcinoma and 17384 controls. Details of the studies are given in Table 1.

Figure 1 shows summary OR in relation to sexual behaviour, reproductive factors, oral contraceptive use and smoking status, based on data from between three and six studies. Both histological types of cervical cancer showed a strong association with the number of sexual partners, with cancer risk increasing with the increasing number of partners. Summary OR (and 95\% CIs) for three or more lifetime partners compared with one partner were $1.94(1.35-2.79)$ for adenocarcinoma and $2.44(1.94-3.07)$ for squamous cell carcinoma. There were no significant differences between the results for adenocarcinoma and for squamous cell carcinoma. Early age at first intercourse was associated with increased risk of both types of cervical cancer, although the association was stronger for squamous cell carcinoma (OR for age at first intercourse of less than 17 years compared with more than 20 years $1.41(0.99-2.00)$ for adenocarcinoma and $2.32(1.89-2.85)$ for squamous cell carcinoma; the difference between these ORs was statistically significant $(P=0.009))$.

Parity was strongly related to the risk of squamous cell carcinoma (summary OR for three or more live births or fullterm pregnancies compared with none $2.71(2.08-3.53)$ ). It was less strongly related to the risk of adenocarcinoma, although there was still a statistically significant association (OR for parity of three or more $1.51(1.02-2.22)$ ), and there appears to be a trend of increasing risk with increasing parity for adenocarcinoma as for squamous cell carcinoma. The difference between the OR for adenocarcinoma and for squamous cell carcinoma in relation to parity of three or more compared to none was statistically

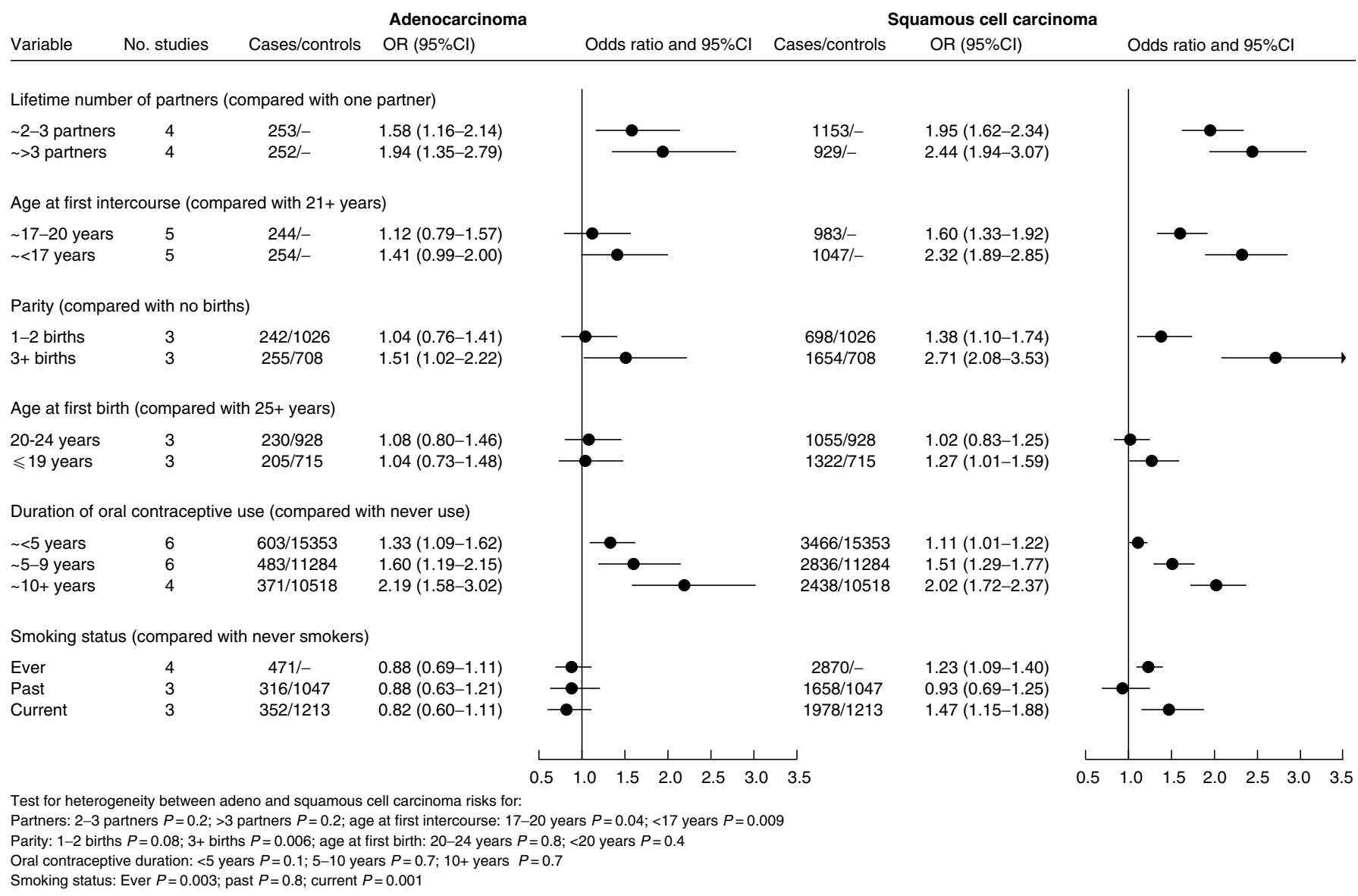

Figure I Summary ORs and $95 \%$ Cls for cervical cancer in relation to sexual behaviour, reproductive factors, oral contraceptive use and smoking status. 


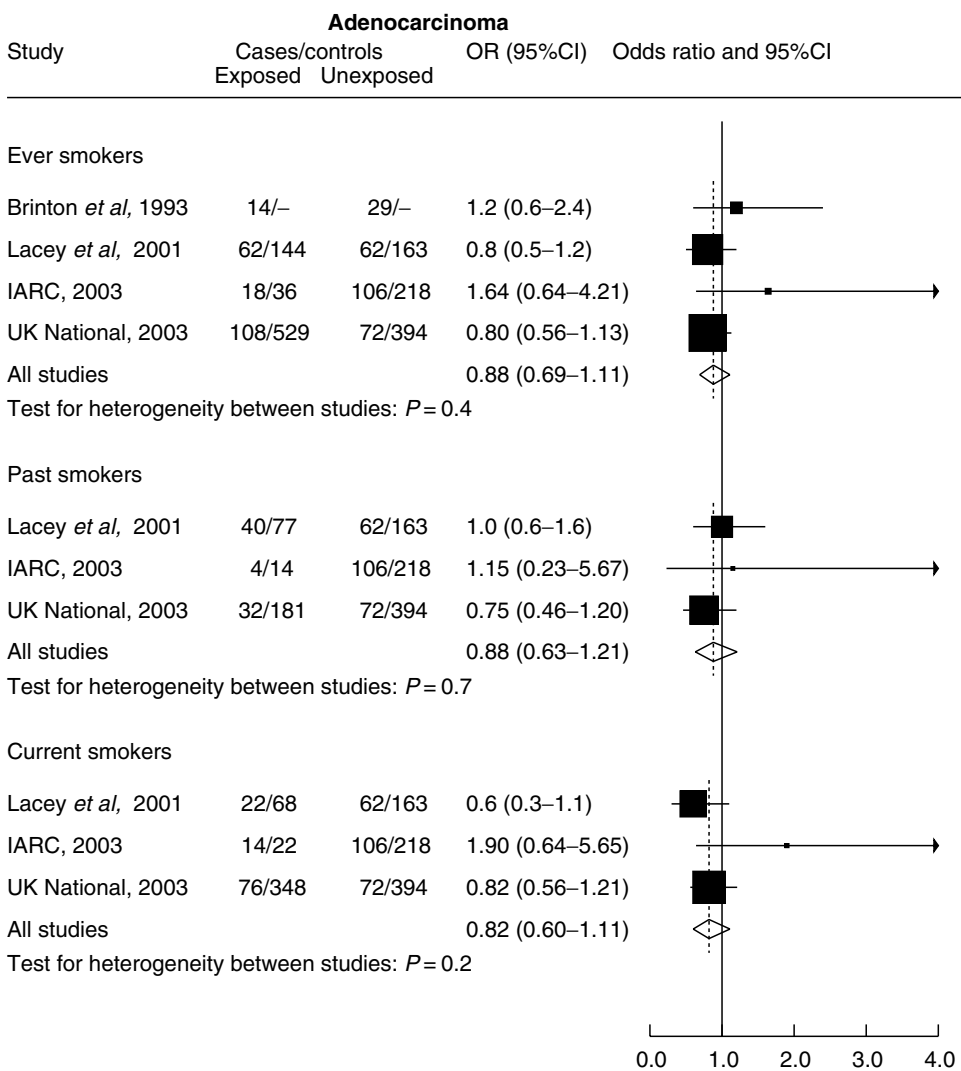

Squamous cell carcinoma

Cases/controls OR $(95 \% \mathrm{Cl}) \quad$ Odds ratio and $95 \% \mathrm{Cl}$

Exposed Unexposed

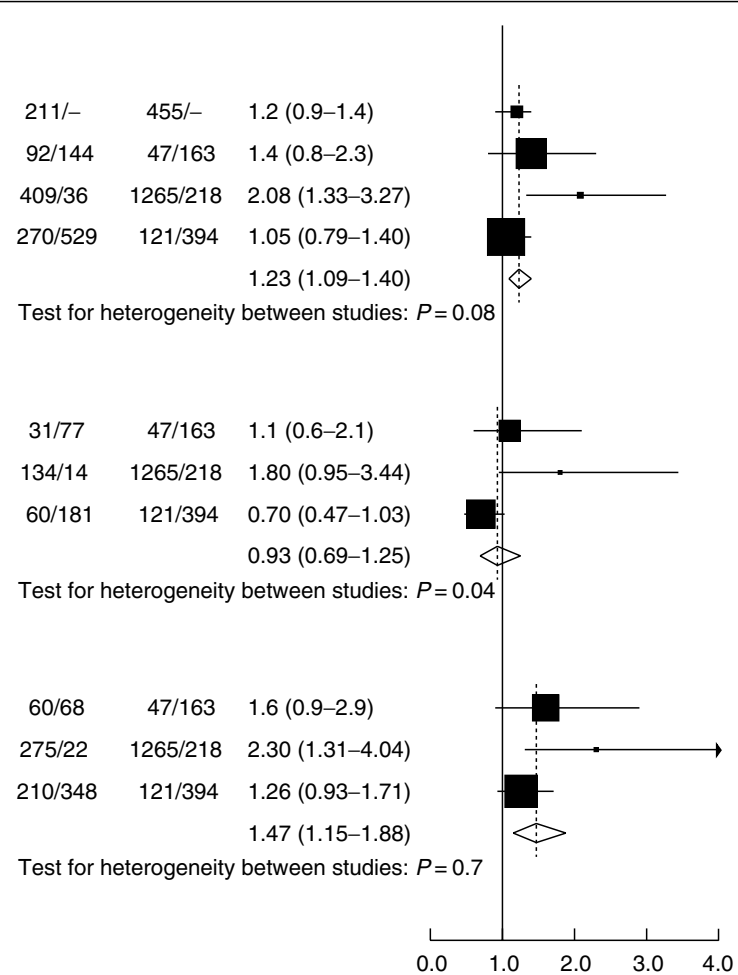

Figure 2 Odds ratios and $95 \% \mathrm{Cls}$ for cervical cancer for ever, past and current smokers vs never smokers.

significant $(P=0.006)$. Age at first birth was not clearly related to either squamous cell or adenocarcinoma of the cervix.

Duration of use of oral contraceptives was strongly related to risk for both adenocarcinoma and squamous cell carcinoma (OR for 10 or more years use compared with never use, 2.19 (1.583.02 ) and $2.02(1.72-2.37)$, respectively), with no significant difference between the results for the two cancer types.

Compared to never smokers, the risk of squamous cell carcinoma was significantly increased in ever smokers (summary $\mathrm{OR}=1.23 \quad(1.09-1.40)$ and in current smokers (summary $\mathrm{OR}=1.47(1.15-1.88)$, although not in past smokers (summary $\mathrm{OR}=0.93(0.69-1.25))$. Adenocarcinoma risk was not associated with smoking status (summary $\mathrm{OR}=0.88(0.69-1.11)$ for ever smokers, $0.82(0.60-1.11)$ for current smokers and $0.88(0.63-$ 1.21) for past smokers compared to never smokers). There was a statistically significant difference between the risks for squamous cell and for adenocarcinoma for ever smoking $(P=0.003)$ and for current smoking $(P=0.001)$.

Statistically significant heterogeneity between studies was present in eight out of the 28 groups of studies $(P$-values for significant heterogeneity between studies: squamous cell carcinoma, $>3$ partners $P=0.002$, parity $1-2 P=0.03$, age at first birth $\leqslant 19$ years $P=<0.0001,<5$ years oral contraceptive use $P=0.03$, past smoking $P=0.04$; adenocarcinoma, parity $1-2 P=<0.0001$, age at first birth $\leqslant 19$ years $P=0.03,<5$ years oral contraceptive use $P=0.04$ ).

The individual study OR for ever, past and current smokers compared to never smokers are shown in Figure 2. There was statistical heterogeneity of marginal significance between individual studies in one group only (squamous cell carcinoma in relation to past smoking; $P=0.04$ ).

Data on smoking intensity were available from two studies only: the summary risk of squamous cell carcinoma increased with increasing intensity of smoking (summary OR $1.22(0.91-1.65)$ and $1.39(1.01-1.91)$ for less than 20 and 20 or more cigarettes per day, respectively, compared to never smokers). The risk of adenocarcinoma was not significantly increased for either group of intensity of smoking compared to never smokers (summary OR $0.80(0.56-1.13)$ and $0.77(0.53-1.13)$ for less than 20 and 20 or more cigarettes per day, respectively.) There was a statistically significant difference between the results for squamous cell and for adenocarcinoma for both levels of intensity (less than 20 cigarettes per day, $P=0.04 ; 20$ or more cigarettes per day, $P=0.01$ ). No heterogeneity between studies was present in any group. Only three studies published results according to duration of smoking, and of these only one (Green et al, 2003) published results for duration of smoking restricted to current smokers. Because of the difference in risk seen for squamous cell cervical cancer between current and past smokers, it was not considered appropriate to combine the results for duration of smoking.

\section{DISCUSSION}

The results of this meta-analysis show consistent qualitative differences between the risks for squamous cell and adenocarcinomas of the cervix in relation to cigarette smoking. Smoking appears to be a risk factor for squamous cell carcinoma, with an increased risk of around 1.5 for current smokers, but not for adenocarcinoma.

The other risk factors investigated did not differ qualitatively between squamous cell and adenocarcinomas; both types of cervical cancer were strongly related to the number of sexual partners and to duration of oral contraceptive use, and both were related to early age at first intercourse and to parity. Neither type of cervical cancer was related to age at first birth in this analysis. 

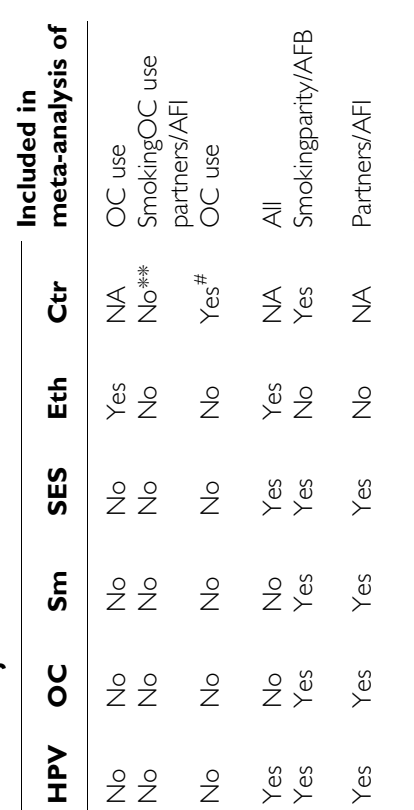

$1 \frac{2-0}{2}$
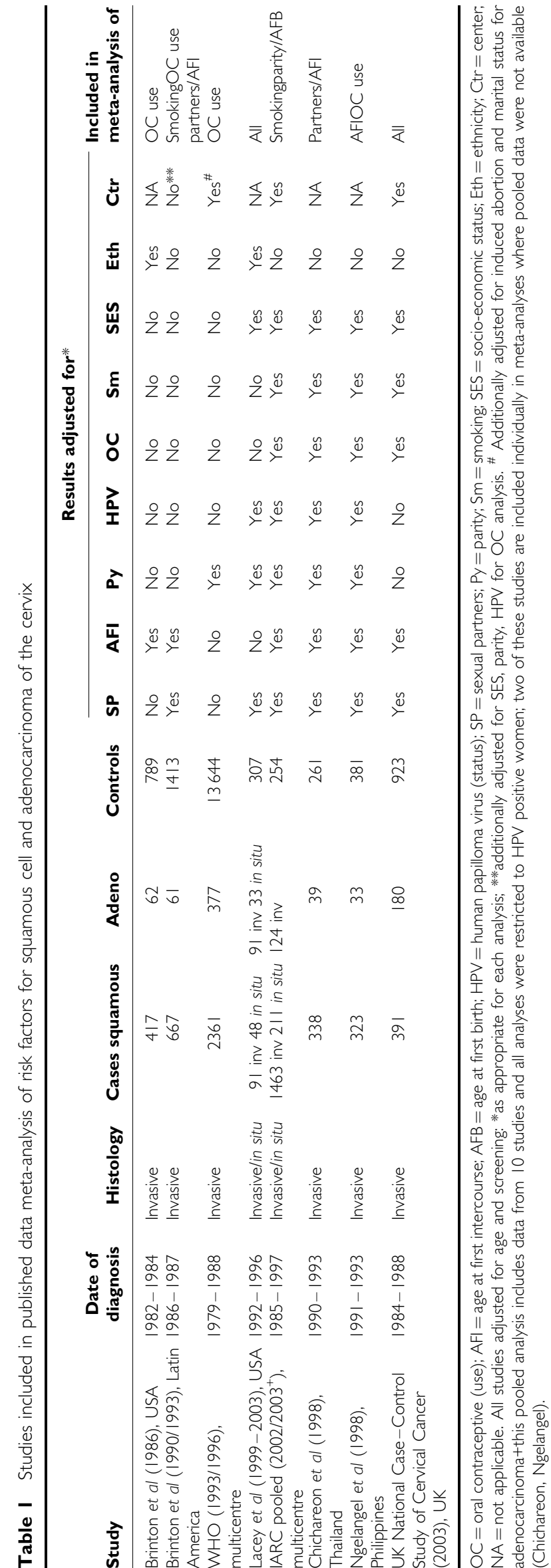

Together with strong evidence that HPV infection is a major, and probably a necessary, causal factor for both squamous cell and adenocarcinoma of the cervix, these findings confirm the impression from recent individual studies that the two main histological types of cervical cancer share the majority of risk factors. The risk factors for cervical adenocarcinoma differ substantially from those for endometrial adenocarcinoma; high parity and the use of oral contraceptives decrease the risk of endometrial cancer, and there is no evidence for an association between endometrial cancer and sexual behaviour or HPV infection (Altekruse et al, 2003; Green et al, 2003; Kjaer and Brinton, 1993).

This meta-analysis of published observational data has a number of limitations (Egger et al, 1998). The most serious is the difference between studies in adjustment for possible confounding factors and for HPV exposure or infection (see Table 1). The four studies included in the smoking meta-analysis, however, all gave results restricted to HPV-positive women (Plummer et al, 2003) or adjusted for HPV status (Lacey et al, 2001) or for lifetime number of sexual partners, a reasonable surrogate for HPV exposure (Brinton et al, 1993; Green et al, 2003). Two studies did not provide results adjusted for HPV infection or exposure (WHO, 1985; Brinton et al, 1986; Thomas and Ray, 1996); both were included only in the meta-analysis of oral contraceptive use, and the results of this analysis were not materially altered when these two studies were omitted. Differences in the risk factor categories used, for example for duration of oral contraceptive use, may also contribute to the statistical heterogeneity seen between studies in some groups. Overall, the number of studies that have published results in a similar way for both squamous cell and adenocarcinoma of the cervix is small, and for some of the analyses the number of studies was very limited. This meant that it was not feasible to investigate heterogeneity between studies formally with respect to different study characteristics. For all of these reasons, the magnitude of the summary odds ratios should be interpreted cautiously.

Observed differences between the risks for squamous cell and for adenocarcinomas could be due to selection or reporting biases, or to differential residual confounding with other risk factors. Cervical screening, for example, is thought to be more effective in detecting squamous cell than adenocarcinomas (Mitchell et al, 1995; Bergstrom et al, 1999); while all studies in this meta-analysis provided results adjusted for screening, the extent of adjustment was variable. However, factors such as these seem unlikely to explain the differences observed in relation to smoking as the two histological types did not differ substantially in the analyses for sexual behaviour, oral contraceptive use or reproductive factors. Some of these factors, such as oral contraceptive use, are known to be related to cervical screening (Eaker et al, 2001). The similarities with respect to other risk factors also suggest that there is unlikely to have been substantial misclassification of cervical adenocarcinomas in these studies (Green et al, 2003).

Some other epithelial cancers, for example those of the nasal cavity, the oesophagus and possibly the lung, appear to show differences between squamous cell and adenocarcinomas in relation to smoking, with the effect of smoking being greater for squamous cell tumours (IARC, 2004, in press). The results of this meta-analysis of available published data suggest that smoking increases the risk of squamous cell carcinoma of the cervix, but has no clear effect on the risk of adenocarcinoma of the cervix. Further studies are needed to confirm this finding.

\section{REFERENCES}

Altekruse SF, Lacey JVJ, Brinton LA, Gravitt PE, Silverberg SG, Barnes WAJ, Greenberg MD, Hadjimichael OC, McGowan L, Mortel R, Schwartz 
PE, Hildesheim A (2003) Comparison of human papillomavirus genotypes, sexual, and reproductive risk factors of cervical adenocarcinoma and squamous cell carcinoma: Northeastern United States. Am J Obstet Gynecol 188: 657-663

Bergstrom R, Sparen P, Adami HO (1999) Trends in cancer of the cervix uteri in Sweden following cytological screening. Br J Cancer 81: 159-166

Berrington A, Cox DR (2003) Generalised least squares for the synthesis of correlated information. Biostatistics 4: 423-431

Brinton LA, Herrero R, Reeves WC, de Britton RC, Gaitan E, Tenorio F (1993) Risk factors for cervical cancer by histology. Gynecol Oncol 51: $301-306$

Brinton LA, Huggins GR, Lehman HF, Mallin K, Savitz DA, Trapido E, Rosenthal J, Hoover R (1986) Long-term use of oral contraceptives and risk of invasive cervical cancer. Int J Cancer 38: 339-344

Brinton LA, Reeves WC, Brenes MM, Herrero R, de Britton RC, Gaitan E, Tenorio F, Garcia M, Rawls WE (1990) Oral contraceptive use and risk of invasive cervical cancer. Int J Epidemiol 19: 4-11

Chichareon S, Herrero R, Munoz N, Bosch FX, Jacobs MV, Deacon J, Santamaria M, Chongsuvivatwong V, Meijer CJ, Walboomers JM (1998) Risk factors for cervical cancer in Thailand: a case-control study. $J$ Natl Cancer Inst 90: $50-57$

Clifford GM, Smith JS, Plummer M, Munoz N, Franceschi S (2003) Human papillomavirus types in invasive cervical cancer worldwide: a metaanalysis. Br J Cancer 88: $63-73$

Cox DR, Snell EJ (1989) Analysis of Binary Data. London: Chapman \& Hall

Eaker S, Adami HO, Sparen P (2001) Reasons women do not attend screening for cervical cancer: a population-based study in Sweden. Prev Med 32: $482-491$

Egger M, Schneider M, Davey SG (1998) Spurious precision? Meta-analysis of observational studies. BMJ 316: 140-144

Green J, Berrington de Gonzalez A, Sweetland S, Beral V, Chilvers C, Crossley B, Deacon J, Hermon C, Jha PK, Mant D, Peto J, Pike MC, Vessey M (2003) Risk factors for adenocarcinoma and squamous cell carcinoma of the cervix in women aged 20-44 years: the UK National Case-Control Study of Cervical Cancer. Br J Cancer 89: 2078-2086

IARC Working Group on the Evaluation of Carcinogenic Risks to Humans 1999 Hormonal Contraception and Post-Menopausal Hormonal Therapy. IARC Monographs on the Evaluation of Carcinogenic Risks to Humans, Vol. 72 Lyon: International Agency for Research on Cancer

IARC 2004 Tobacco Smoke and Involuntary Smoking. IARC Monographs on the Evaluation of Carcinogenic Risks to Humans, Vol. 83 Lyon: International Agency for Research on Cancer (in press)
Kjaer SK, Brinton LA (1993) Adenocarcinomas of the uterine cervix: the epidemiology of an increasing problem. Epidemiol Rev 15: 486-498

Lacey JV, Brinton L, Abbas FM, Barnes WA, Gravitt PE, Greenberg MD, Greene SM, Hadjimichael O, McGowan L, Mortel R, Schwartz PE, Silverberg SG, Hildesheim A (1999) Oral contraceptives as risk factors for cervical adenocarcinomas and squamous cell carcinomas. Cancer Epidemiol Biomarkers Prev 8: 1079-1985

Lacey JVJ, Frisch M, Brinton LA, Abbas FM, Barnes WA, Gravitt PE, Greenberg MD, Greene SM, Hadjimichael OC, McGowan L, Mortel R, Schwartz PE, Zaino RJ, Hildesheim A (2001) Associations between smoking and adenocarcinomas and squamous cell carcinomas of the uterine cervix (United States). Cancer Causes Control 12: 153-161

Mitchell H, Medley G, Gordon I, Giles G (1995) Cervical cytology reported as negative and risk of adenocarcinoma of the cervix: no strong evidence of benefit. Br J Cancer 71: 894-897

Munoz N, Franceschi S, Bosetti C, Moreno V, Smith JS, Shah KV, Meijer CJ, Bosch F (2002) Role of parity and human papillomavirus in cervical cancer: the IARC multicentric case-control study. Lancet 359: 1093 1101

Ngelangel C, Munoz N, Bosch FX, Limson GM, Festin MR, Deacon J, Jacobs MV, Santamaria M, Meijer CJ, Walboomers JM (1998) Causes of cervical cancer in the Philippines: a case-control study. J Natl Cancer Inst 90: 43-49

Parazzini F, La Vecchia C (1990) Epidemiology of adenocarcinoma of the cervix. Gynecol Oncol 39: 40-46

Plummer M, Herrero R, Franceschi S, Meijer CJ, Snijders PJ, Bosch FX, de Sanjose S, Munoz N (2003) Smoking and cervical cancer: pooled analysis of the IARC multi-centric case-control study. Cancer Causes Control 14: $805-814$

Sasieni P, Adams J (2001) Changing rates of adenocarcinoma and adenosquamous carcinoma of the cervix in England. Lancet 357: 1490 - 1493

Thomas DB, Ray RM (1996) Oral contraceptives and invasive adenocarcinomas and adenosquamous carcinomas of the uterine cervix. The World Health Organization Collaborative Study of Neoplasia and Steroid Contraceptives. Am J Epidemiol 144: 281-289

Walboomers JM, Jacobs MV, Manos M, Bosch F, Kummer A, Shah K, Snijders PJ, Peto J, Meijer CJ, Munoz N (1999) Human papillomavirus is a necessary cause of invasive cervical cancer worldwide. J Pathol 189: $12-19$

WHO (1985) Invasive cervical cancer and combined oral contraceptives. WHO collaborative study of neoplasia and steroid contraceptives. $\mathrm{Br}$ Med J Clin Res Ed 290: $961-965$ 